\title{
Investigation of microRNAs in mouse macrophage responses to lipopolysaccharide- stimulation by combining gene expression with microRNA-target information
}

\author{
Chia-Chun Chiu, Wei-Sheng Wu*
}

From Joint 26th Genome Informatics Workshop and Asia Pacific Bioinformatics Network (APBioNet) 14th International Conference on Bioinformatics (GIW/InCoB2015)

Tokyo, Japan. 9-11 September 2015

\begin{abstract}
Background: Toll-like receptors, which stimulated by pathogen-associated molecular patterns such as lipopolysaccharides (LPS), induces the releasing of many kinds of proinflammatory cytokines to activate subsequent immune responses. Plenty of studies have also indicated the importance of TLR-signalling on the avoidance of excessive inflammation, tissue repairing and the return to homeostasis after infection and tissue injury. The significance of TLR-signalling attracts many attentions on the regulatory mechanisms since several years ago. However, as newly discovered regulators, how and how many different microRNAs (miRNAs) regulate TLRsignalling pathway are still unclear.

Results: By integrating several microarray datasets and miRNA-target information datasets, we identified 431 miRNAs and 498 differentially expressed target genes in bone marrow-derived macrophages (BMDMs) with LPSstimulation. Cooperative miRNA network were constructed by calculating targets overlap scores, and a sub-network finding algorithm was used to identify cooperative miRNA modules. Finally, 17 and 8 modules are identified in the cooperative miRNA networks composed of miRNAs up-regulate and down-regulate genes, respectively.

Conclusions: We used gene expression data of mouse macrophage stimulated by LPS and miRNA-target information to infer the regulatory mechanism of miRNAs on LPS-induced signalling pathway. Also, our results suggest that miRNAs can be important regulators of LPS-induced innate immune response in BMDMs.
\end{abstract}

\section{Background}

The immune system is composed of innate and adaptive immune systems, in which the machinery behind innate immune system is very ingenious due to the conserved ability to recognize microbial components during evolution process. Toll-like receptors (TLRs) are a kind of specialized sensor responsible for recognizing molecular patterns of pathogens and bridging innate and adaptive immunity by activation of antigen-presenting cells such as dendritic cells and macrophages. Upon exposure of

\footnotetext{
* Correspondence: wessonwu@mail.ncku.edu.tw

Department of Electrical Engineering, National Cheng Kung University, No.1 University Road, Room 92879, 701 Tainan, Taiwan
}

molecular patterns of pathogens, TLRs initiate a cascade of intracellular signalling transduction that leads to secretion of inflammatory mediators, including cytokines and chemokines, inducing expression changes of a broad spectrum of genes that regulate defense function against pathogens.

TLR4, the best characterized member of TLR family, can detect lipopolysaccharide (LPS) from Gram-negative bacteria. Once TLR4 is stimulated by LPS, two independent signalling pathways (TRIF-dependent and MyD88-dependent) will be initiated, and many import transcription factors (TNF- $\alpha$, IFN- $\beta$, IL- $1 \beta$, etc.) will be activated as well. Although several mechanisms which 
are responsible for the regulation of TLR4-induced signalling pathways have been already well-studied, including physical interactions, changes of conformation, phosphorylation, ubiquitylation, and etc [1-3], how an anti-inflammatory response is induced and the processes by which inflammation remain unclear [4]. Among these regulators, miRNAs have drew enough notice as a newly identified regulators in last several years.

MicroRNAs (miRNAs), a class of small non-coding RNAs, has emerged as important regulators in the development of immune and inflammatory responses [4-8]. The importance of miRNAs in the modulation of normal and pathological immune function has been shown in multiple studies in which deregulation of miRNAs was demonstrated to accompany diseases associated with excessive or uncontrolled inflammation. As a critical part of regulatory networks in innate immunity, the dysregulation of some specific miRNAs would modulate the regulation of inflammatory in stimulated immune cells. According to the literature [9], many miRNAs are reported to be dys-regulated in activated immune cells, such as miR-146a.

Recently, many studies have attempted to develop methods to understand miRNA co-operativity. Most of these studies however did not actually considered coexpression profiles of mRNAs and miRNAs. Considering that most miRNAs exert their functions through interactions with other miRNAs, an understanding of a miRNA network context using both co-expression pattern and the sequence complementarity between miRNAs and mRNAs is essential to discover the cooperative regulation of miRNAs.

In this paper, we used two kinds of data sets to infer the regulatory mechanism of miRNAs on LPS-induced signalling pathway. mRNA expression data and miRNA-target information data are used to identify reliable regulatory relationship between miRNAs and mRNA, and then cooperative miRNAs can be identified using hypergeometric test. Besides, all differentially expressed target genes were used to perform coherent functional analysis, the possible function of miRNAs which actually regulate gene expression could be hence inferred. Using these cooperative miRNAs and their common target genes, a cooperative miRNA network could be constructed and the cooperative miRNA modules could be also identified.

\section{Materials and methods Overview}

The work flow for identification of co-regulating miRNAs in mouse macrophages with stimulation of LPS. Microarray expression data and miRNA-target information data are used to identify reliable regulatory relationship between miRNAs and mRNA, and then cooperative microRNAs can be identified using hypergeometric test. Besides, all differentially expressed target genes were used to perform coherent functional analyses, the possible function of miRNAs which actually regulate gene expression could be hence inferred. Using these cooperative miRNAs and their common target genes, a cooperative miRNA network could be constructed and the cooperative miRNA modules could be also identified.

\section{Datasets}

In this study, five expression profile datasets and four miRNA-target information datasets are used. Expression datasets, which were downloaded from NCBI GEO database [10], are from five experiments generated individually by Barish et al [11], El Chartouni et al [12], Ghigo et al [13], Mages et al [14] and Millien et al [15], which were downloaded from NCBI GEO database [10]. Barish et al used Illumina MouseWG-6 v2.0 expression beadchip to compare the changes of gene expression between wildtype and Bcl6 $\mathrm{KO}$ bone marrow-derived macrophages of mouse in the absence or presence of LPS (GSE24792). El Chartouni et al used Agilent014868 Whole Mouse Genome Microarray to study TLR4 induced transcriptional responses in interleukin 4 primed mouse bone marrow-derived macrophages (GSE21895). Ghigo et al used Agilent-014868 Whole Mouse Genome Microarray to compare the difference of gene expression of mouse bone marrow-derived macrophage response to T. whipplei and LPS (GSE20210). Magges et al used Affymetrix Mouse Genome 4302.0 Array to study the development of a state of refractoriness to a second stimulation in mouse bone marrow-derived macrophages treated with LPS (GSE8621). Millien et al used Illumina MouseWG-6 v2.0 expression beadchip to study the effects of mouse bone marrow derived macrophages treated with PAO, IFN-gamma, or LPS (GSE48609). On the other hand, miRNA-target gene interaction datasets were downloaded from Mouse Genome Informatics (MGI) database [16]. These datasets mainly come from four different data sources, in which miRTarBase [17] data are validated, but microt-cds [18], miRDB [19] and Pictar [20] data are all predicted. The list of mouse genetic markers which also downloaded from MGI database was used as the list of all genes and miRNA in mouse.

\section{Expression data preprocessing and differentially expressed genes identification}

In each expression dataset, only wild-type samples in the absence or presence of LPS were used in subsequent data analyses. The value distribution service of GEO2R in NCBI GEO database was used to determined if this data are normalized and cross-comparable, and the results revealed that only the datasets from Barish et al study and from Millien et al study are not needed to be normalized. Other three datasets were quantile-normalized using R package "affyPLM" [21]. For every expression 
datasets, differential expression analyses were performed using R package "limma" [22]. Benjamini-Hochberg method was used to control the false discovery rate (FDR), and genes with adjusted p-value less than 0.05 were identified as differentially expressed (DE) genes.

\section{Preprocessing of miRNA-target gene interaction data}

Due to a large part of miRNA-target gene interaction data consists of predicted data, there are many false positives in these datasets. Therefore, only the data from miRTarBase and the data appear at least in two of three predicted data sources were retained, and then we obtained a list with 822697 miRNA-target gene interaction data. We obtained 712 miRNAs from this list, and then remove the miRNAs which could be found in the list of mouse genetic markers. Finally, 703 miRNAs were retained.

\section{Gene set enrichment analysis}

We carried out the gene set enrichment analysis to identify the miRNAs which actually participate in the regulation of target genes. For each miRNA, a $2 \times 2$ contingency table was built (whether the genes are targets of this miRNA or not versus whether the genes are differentially expressed) to access the enrichment level using Fisher's exact test. After all miRNAs were tested, Benjamini-Hochberg procedure for multiple test correction was used to correct p-values. This miRNA would be considered to have a significant number of differentially expressed target genes if the adjusted p-value of Fisher's exact test is less than 0.05, and this miRNA could be thus considered a reliable regulator in mouse macrophages under LPS-stimulated condition.

\section{Coherent functional analysis}

Cytoscape v3.2.1 (http://cytoscape.org/) with ClueGO v2.1.6 plug-in [23] was used to perform GO and KEGG pathway analyses. ClueGO let us to obtain the distribution of the list of differentially expressed target genes across the GO terms and pathways. We used right-sided hypergeometric test to calculate enrichment level and used Benjamini-Hochberg adjustment to correct p-values in multiple test. A GO term or pathway would be considered to be enriched in differentially expressed target genes if its adjusted p-value is less than 0.05 . We analyzed up-regulated target genes and down-regulated target genes using these settings in GO and KEGG pathway analyses, respectively.

\section{Construct cooperative miRNA network}

Cooperative miRNA network (CMN) is defined as a network composed of cooperative miRNAs sharing a significant number of differentially expressed target genes, which could be used to grouping these cooperative miRNAs. The assumption behind $\mathrm{CMN}$ is based on the concepts of co-expressed and co-regulation, which means that if two regulators are co-operative, the two regulators should share a significant number of common target genes with similar expression profiles. In a CMN, each node represents a specific miRNA and each edge represents a specific co-operativity between a pair of miRNAs. We performed significance analysis using hypergeometric test as follows:

$$
\mathrm{P}_{\text {overlap }}=\sum_{k=m}^{\min \left(N_{i}, N_{j}\right)} \frac{C_{k}^{N_{i}} C_{N_{j}-k}^{N-N_{i}}}{C_{N_{j}}^{N}}
$$

where $N_{i}$ and $N_{j}$ are the number of differentially expressed target genes of miRNA $i$ and of miRNA $j$, respectively. $P_{\text {overlap }}$ is the p-value on this test, $N$ is the total number of DE genes and $m$ is the number of common differentially expressed target genes between miRNA $i$ and miRNA $j$. Subsequently, Benjamini-Hochberg method was used to adjust $\mathrm{p}$-values and the miRNA pair with adjusted p-value less than 0.01 was considered as sharing a significant number of common differentially expressed target genes. After these significance analyses, a list of cooperative miR-NAs would be obtained. Next step, a target overlap score was used to represent the weight of each edge in the CMN by calculating the Jaccard similarity coefficient. The $P_{\text {overlap }}$ or $-\log P_{\text {overlap }}$ value were not used as overlap scores due to the large difference between these p-values. The target overlap score according to the definition in Na and Kim's paper [24] is defined as follows:

$$
\mathrm{S}_{\text {overlap }}= \begin{cases}\frac{|\operatorname{Targets}(i) \cap \operatorname{Targets}(j)|}{|\operatorname{Targets}(i) \cup \operatorname{Targets}(j)|}, & i \neq j \\ 1 & , i=j\end{cases}
$$

, where Targets $(i)$ and Targets $(j)$ represent the set of target genes of miRNA $i$ and miRNA $j$, respectively. As a result, the list of cooperative miRNAs and the target overlap scores were used to construct CMN.

\section{Extraction of cooperative miRNA modules (CMM)}

In this paper, a sub-network detection algorithm, Molecular COmplex DEtection algorithm (MCODE) [25], was applied to find possible coherent modules in the global cooperative miRNA network. The MCODE, which is a clustering algorithm based on graph theorem, is specifically designed to find complexes by identifying densely connected subgraphs in networks. MCODE algorithm is composed of three stages: vertex weighting, complex prediction and an optional post-processing step. The core clustering coefficient is used as the weights of nodes. Once the weights are computed, the algorithm traverses the weighted graph using greedy algorithm to isolate densely connected regions. The post-processing step filters or adds nodes 
based on connectivity criteria. MCODE has a parameter that specifies the size of clusters returned. All parameters in MCODE are applied with default values. Sub-networks are filtered if they do not contain at least a 2-core (graph of minimum degree 2). This approach allows us to assign one miRNA to multiple clusters, considering a biological principle that miRNAs can have multiple functions.

\section{Results}

\section{Differentially expressed mRNA}

After differential expression analyses, we obtained 8990 DE genes from GSE20210 dataset, in which 3532 genes are up-regulated and 5458 genes are down-regulated. Similarly, there are 4514 DE genes (including 1910 upregulated genes and 2604 down-regulated genes) identified in GSE21895 dataset, 4597 DE genes (including 2094 up-regulated genes and 2863 down-regulated genes) identified in GSE24792 data set, 2880 DE genes (including 1360 up-regulated genes and 1520 down-regulated genes) identified in GSE48609 dataset, and 6516 DE genes (including 2523 up-regulated genes and 3633 down-regulated genes) identified in GSE8621 dataset. In order to avoid the problems caused by bias between different platforms, we only used the intersection of the results from these five expression datasets, which cause that 233 up-regulated genes and 286 down-regulated genes left (see Figure 1). Besides, any differentially expressed probe from any platform which could not be mapped to any Entrez gene ID or any gene does not appear in the list of miRNA-target gene interaction were removed. Finally, 228 up-regulated genes and 270 downregulated genes were retained for subsequent analyses.

\section{GO and KEGG enrichment of differentially expressed genes}

In this study, we performed KEGG-enrichment analysis and GO-enrichment on differentially expressed genes separately. For down-regulated genes, they enrich in two different pathways: antigen-receptor and $B$ cell receptor signalling pathways (see Figure 2a). Besides, we found that these down-regulated genes enrich in several immune system processes, including adaptive immune response based on somatic recombination of immune receptors built from immunoglobulin super-family domains, positive regulation of immune effector process, toll-like receptor 4 pathways, regulation of immunoglobulin production, regulation of toll-like receptor signalling pathway, negative regulation of immune effector process, regulation of leukocyte mediated immunity and so on (see Figure $2 \mathrm{~b}$ ). On the other hand, seven different functional pathways were found enriched in up-regulated genes, including $\beta$-Alanine metabolism, fructose and mannose metabolism, N-glycan biosynthsis, Fanconi anemia pathway, MAPK cascade, p38 MAPK signalling pathway and FAS pathway/Stress induction of HSP regulation (see Figure 3a). After GO-enrichment analysis, 10 immune system process were found enriched in up-regulated genes, including Adipocytokine signaling pathway, NF- $\kappa \mathrm{B}$ signalling pathway, Hepatitis B, TNF signalling pathway, Rap1 signalling pathway, Chemokine signalling pathway, transcriptional mis-regulation in cancer, Cholinergic synapse, osteoclast differentiation and toll-like receptor signalling pathway (see Figure 3b).

\section{Construction and characterization of cooperative miRNA networks}

Figure 4 shows the global CMN created by superimposing CMN composed of miR

NAs up-regulating target genes and CMN composed of miRNAs down-regulating target genes, there exists 417 nodes and 7901 edges. In CMN, each node corresponds to a miRNA that has a significant number of differentially expressed target genes in mouse macrophage with the stimulation of LPS, and each edge represents that the miRNA pair on both side of this edge share a significant number of common differentially expressed target genes. The nodes with yellow colour are the miRNAs which only up-regulate genes, the nodes with red colour are the miRNAs which only down-regulate genes, and the nodes with blue colour are the miRNAs which up-regulate and down-regulate genes, which implies that there may exists a more complex mechanism behind the regulation of

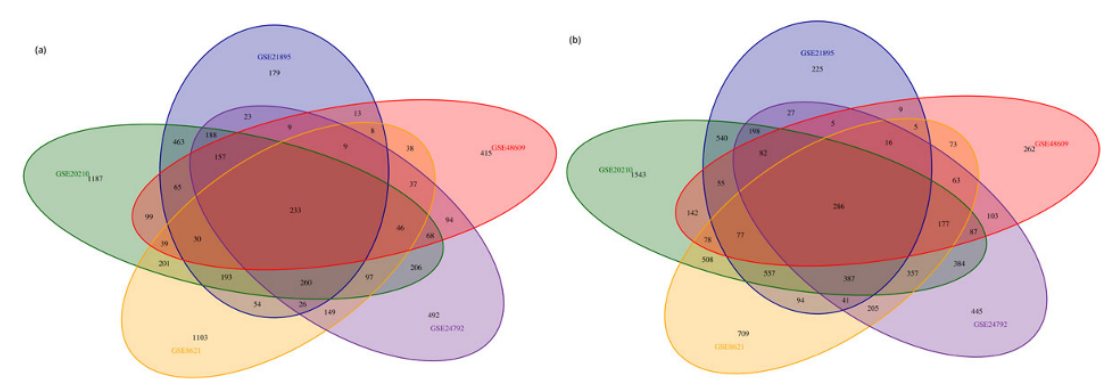

Figure 1 The intersection of up-regulated and down-regulated DE genes among five expression datasets. 
(a)

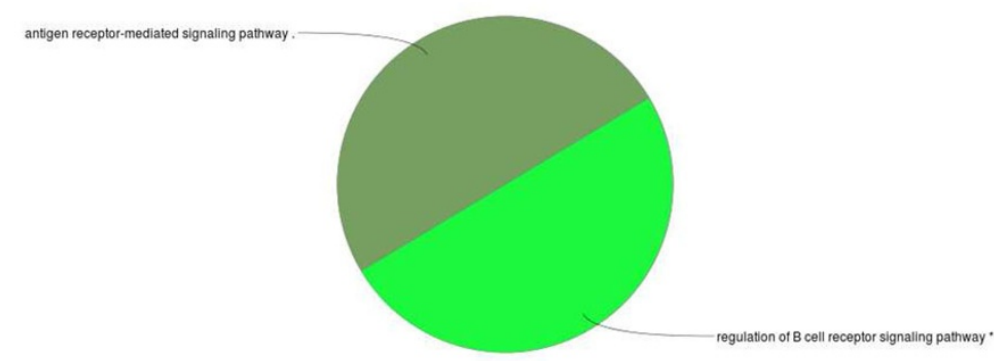

(b)

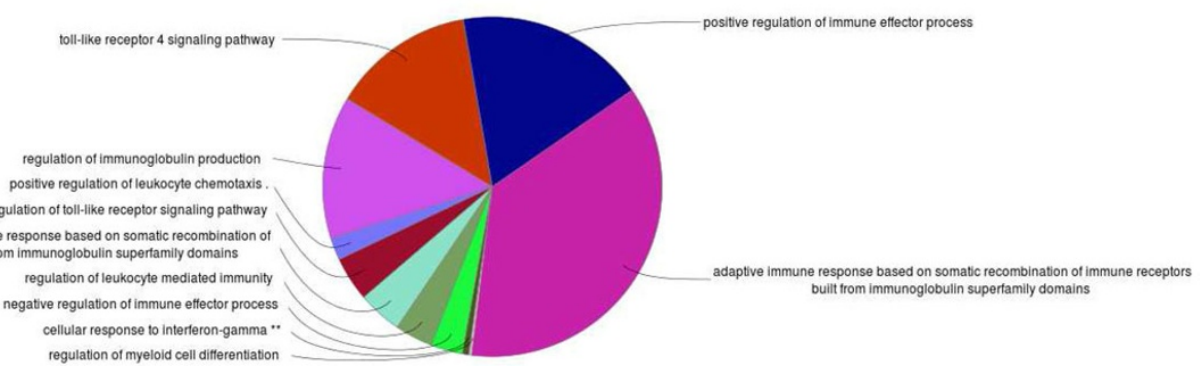

Figure 2 The results of GO enrichment analyses on DE genes. (a) The number of enriched GO terms in down-regulated genes is 6, and these GO terms can be classified into two classes. (b) The number of enriched GO terns in up-regulated genes is 152, and these terms can be classified into 11 class.

(a)

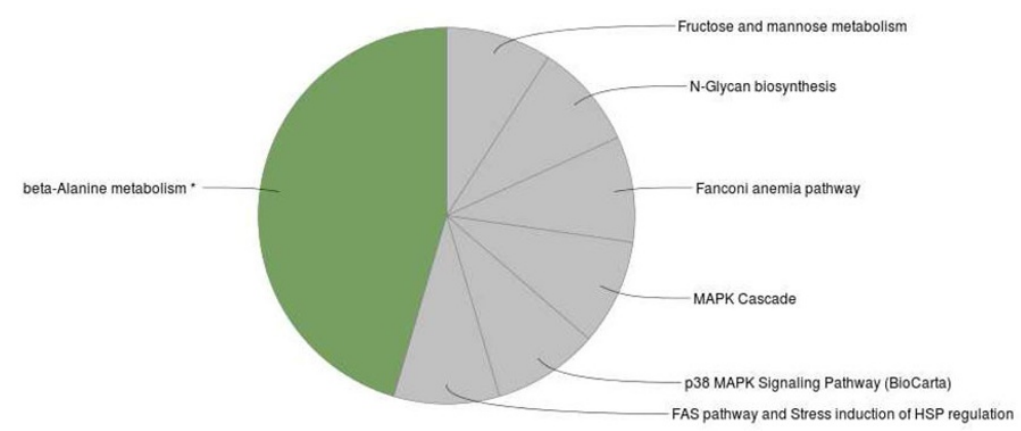

(b)

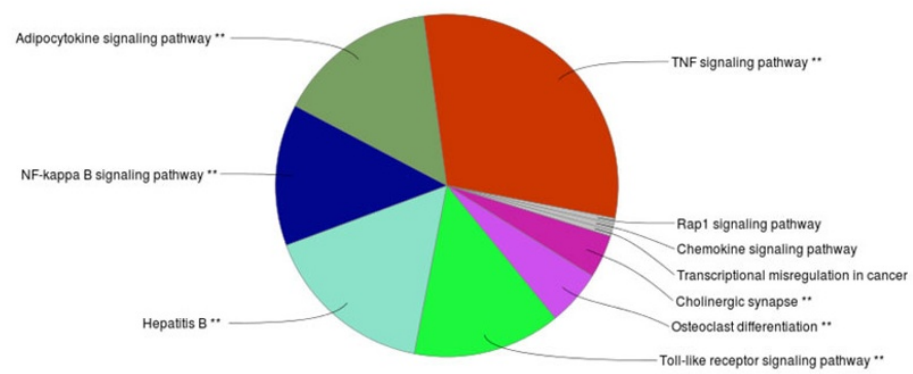

Figure 3 The results of KEGG enrichment analyses on DE genes. (a) The number of enriched KEGG pathways in down-regulated genes is 11 , and (b) the number of enriched KEGG pathways in up-regulated genes is 78. 


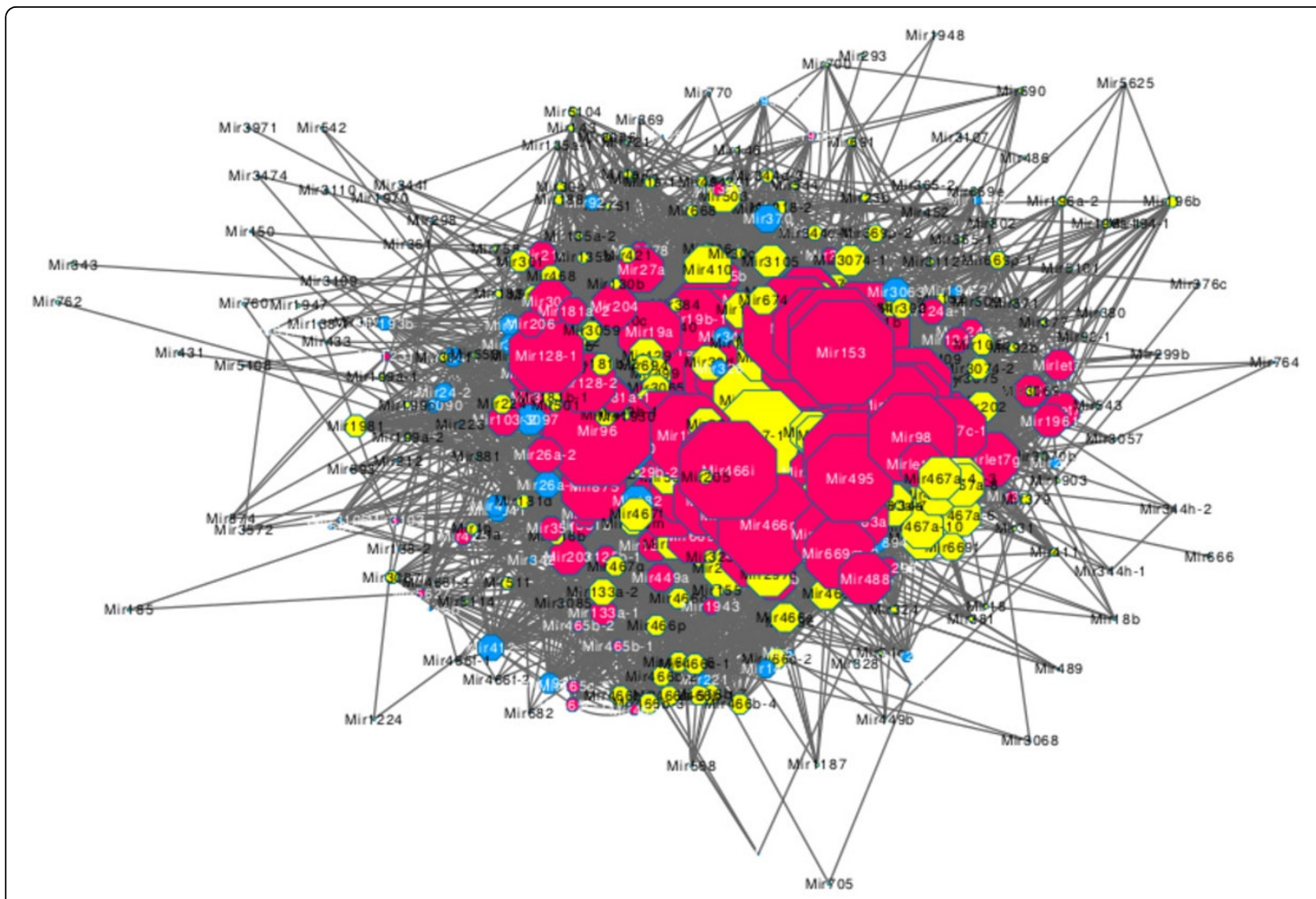

Figure 4 The superposition of CMNs which composed of microRNAs regulating up-regulated genes and down-regulated genes.

innate immunity. Using MCODE on CMN composed of miRNAs up-regulating target genes, 17 cooperative miRNA modules were found (Table 1). The numbers of miRNAs and connections of CMMs range from 3 to 43 and 3 to 696, respectively. While using MCODE on
CMN composed of miRNAs down-regulating target genes, 8 cooperative miRNA modules were found (Table 2) and the numbers of miRNAs and connections of CMMs range from 3 to 34 and 3 to 374, respectively. This result shows that most of miRNAs are multiple connected, and most of

Table 1 Cooperative miRNA modules in which miRNAs up-regulate genes.

\begin{tabular}{|c|c|c|c|c|}
\hline Cluster & Score & Nodes & Edges & miRNAs \\
\hline 1 & 33.143 & 43 & 696 & $\begin{array}{l}\text { Mir106b, Mirlet7b, Mir98, Mir297c, Mir9-1, Mir9-2, Mir200b, Mir467d, Mir9-3, Mir669f, Mir467c, Mir466n, Mir297a-4, } \\
\text { Mir669m-2, Mir669m-1, Mir302a, Mir467b, Mirlet7a-1, Mir200a, Mir141, Mir297a-3, Mir669l, Mirlet7c-2, Mirlet7f-1, } \\
\text { Mir467a-10, Mir467f, Mir467a-9, Mir669d-2, Mir669d, Mir467e, Mir669b, Mir467a-4, Mir93, Mir467a-3, Mir467a-1, } \\
\text { Mir467a-2, Mir297b, Mir302d, Mir17, Mir467a-8, Mir467a-7, Mir467a-6, Mir467a-5 }\end{array}$ \\
\hline 2 & 14.067 & 31 & 211 & $\begin{array}{l}\text { Mir294, Mir295, Mir302c, Mir466c-2, Mir466c-1, Mir466p, Mir291b, Mir291a, Mir466, Mir466e, Mir467g, Mir466b-4, } \\
\text { Mir466m, Mir292, Mir466b-3, Mir466b-7, Mir466b-6, Mir466b-5, Mir466b-8, Mir539, Mir493, Mir466o, Mir106a, } \\
\text { Mir20bm Mir302b }\end{array}$ \\
\hline 3 & 9.4 & 21 & 94 & $\begin{array}{l}\text { Mir20a, Mir153, Mir202, Mir322, Mirlet7i, Mirlet7c-1, Mir466i, Mirlet7e, Mir7b, Mir22, Mir7-2, Mirlet7f-2, Mir1961, } \\
\text { Mirlet7d, Mir488, Mir19b-1, Mir320, Mir669p-1, Mir196b, Mir669p-2, Mirlet7a-2 }\end{array}$ \\
\hline 4 & 8.952 & 43 & 188 & $\begin{array}{l}\text { Mir503, Mir216a, Mir883a, Mir421, Mir883b, Mir551b, Mir5619, Mir30c-2, Mir3065, Mir3069, Mir410, Mir128-2, Mir5624 } \\
\text { Mir103-1, Mir742, Mir344e, Mir344d-2, Mir133a-2, Mir3074-1, Mir181b-2, Mir133a-1, Mir499, Mir743, Mir497, Mir3082, } \\
\text { Mir300, Mir344d-1, Mir344d-3, Mir124a-3, Mir30b, Mir124a-1, Mir124a-2, Mir495, Mir448, Mir101a, Mir188, Mir30c-1, } \\
\text { Mir27b, Mir382, Mir301b, Mir374c, Mir323, Mir384 }\end{array}$ \\
\hline 5 & 7.29 & 32 & 113 & $\begin{array}{l}\text { Mir494, Mir429, Mir15b, Mir128-1, Mir5616, Mir1907, Mir107, Mir30a, Mir33, Mir103-2, Mir140, Mir222, Mir181b-1, } \\
\text { Mir3074-2, Mir7-1, Mir216b, Mir181d, Mir16-1, Mir19b-2, Mir468, Mir15a, Mir743b, Mir96, Mir181c, Mir1912, Mir214, } \\
\text { Mir674, Mir1906-1, Mir582, Mir27a, Mir1906-2, Mir301 }\end{array}$ \\
\hline 6 & 5.167 & 25 & 62 & $\begin{array}{l}\text { Mir465b-1, Mir152, Mir32, Mir367, Mir465, Mir218-1, Mir465c-2, Mir465c-1, Mir758, Mir129-1, Mir30d, Mir129-2, } \\
\text { Mir3066, Mir706, Mir204, Mir669c, Mir19a, Mir186, Mir465b-2, Mir1192, Mir875, Mir92b, Mir16-2, Mir669h, Mir673 }\end{array}$ \\
\hline
\end{tabular}


Table 1 Cooperative miRNA modules in which miRNAs up-regulate genes. (Continued)

\begin{tabular}{lllll}
\hline 7 & 4 & 4 & 6 & Mir694, Mir199a-2, Mir199a-1, Mir199b \\
\hline 8 & 3.333 & 4 & 5 & Mir212, Mir351, Mir125b-1, Mir125b-2 \\
\hline 9 & 3 & 13 & 18 & Mir449a, Mir218-2, Mir449b, Mir143, Mir1930, Mir3059, Mir34b, Mir5104, Mir23b, Mir211, Mir23a, Mir672, Mir224 \\
\hline 10 & 3 & 3 & 3 & Mir466f-2, Mir466f-1, Mir466f-3 \\
\hline 11 & 3 & 3 & 3 & Mir29a, Mir29b-1, Mir29c \\
\hline 12 & 3 & 3 & 3 & Mir1981, Mir340, Mir142 \\
\hline 13 & 3 & 3 & 3 & Mir1a-2, Mir148a, Mir148b \\
\hline 14 & 3 & 3 & 3 & Mir3107, Mir486, Mir326 \\
\hline 15 & 3 & 3 & 3 & Mir544, Mir377, Mir1903 \\
\hline 16 & 3 & 3 & 3 & Mir692-3, Mir692-1, Mir692-2 \\
\hline 17 & 3 & 3 & 3 & Mir149, Mir3085, Mir5627 \\
\hline
\end{tabular}

Cluster Score Nodes Edges miRNAs

Table 2 Cooperative miRNA modules in which miRNAs down-regulate genes.

\begin{tabular}{lllll}
\hline Cluster & Score & Nodes & Edges & miRNAs \\
\hline 1 & 22.667 & 34 & 374 & $\begin{array}{l}\text { Mir129-1, Mir19a, Mirlet7i, Mir466l, Mir1912, Mirlet7e, Mir19b-2, Mir1961, Mir98, Mir350, Mir466n, Mirlet7d, Mir129-2, } \\
\text { Mir1192, Mirlet7f-1, Mirlet7c-1, Mirlet7a-1, Mirlet7a-2, Mir96, Mirlet7c-2, Mir669m-2, Mir669m-1, Mir291a, Mir7-1, } \\
\text { Mir497, Mir335, Mirlet7f-2, Mir19b-1, Mir204, Mir875, Mir883a, Mirlet7g, Mir743b, Mir743 }\end{array}$ \\
\hline 2 & 14.364 & 34 & 237 & $\begin{array}{l}\text { Mir351, Mir5615-2, Mir93, Mirlet7b, Mir876, Mir470, Mir17, Mir677, Mir1983, Mir3063, Mir9-2, Mir320, Mir495, Mir9-3, } \\
\text { Mir203, Mir182, Mir672, Mir449a, Mir488, Mir26a-2, Mir9-1, Mir330, Mir669k, Mir20b, Mir106a, Mir128-1, Mir466d, } \\
\text { Mir466i, Mir218-1, Mir539, Mir883b, Mir153, Mir20a, Mir292 }\end{array}$ \\
\hline 3 & 5 & 5 & 10 & Mir465c-1, Mir465c-2, Mir465, Mir465b-2, Mir465b-1 \\
\hline 4 & 4.75 & 17 & 38 & $\begin{array}{l}\text { Mir362, Mir128-2, Mir147, Mir338, Mir412, Mir125a, Mir322, Mir1941, Mir200b, Mir125b-2, Mir3097, Mir34b, Mir370, } \\
\text { Mir125b-1, Mir133a-1, Mir133a-2, Mir1298 }\end{array}$ \\
\hline 5 & 4.5 & 5 & 9 & Mir124a-3, Mir5624, Mir124a-1, Mir673, Mir124a-2 \\
\hline 6 & 3.333 & 4 & 5 & Mir667, Mir103-2, Mir3078, Mir107 \\
\hline 7 & 3.2 & 6 & 8 & Mir5627, Mir3090, Mir5623, Mir346, Mir3087, Mir3113 \\
\hline 8 & 3 & 3 & 3 & Mir24-2, Mir149, Mir24-1 \\
\hline Cluster Score Nodes Edges miRnAs &
\end{tabular}

Cluster Score Nodes Edges miRNAs

miRNAs do not own significantly more edges than others, which means that most of miRNAs must act co-operatively.

\section{Conclusions}

LPS-induced signalling must be tightly regulated to avoid excessive inflammation and to allow for tissue repair and the return to homeostasis after infection and tissue injury. Though miRNAs are critical regulators of immune responses, whether they are involved in LPS-induced signalling pathway and how is their expression regulated in mouse macrophages are still unclear. We combine mRNA expression data and miRNA-target information to infer the regulatory mechanism of miRNAs on LPS-induced signalling pathway. Also, our results suggest that miRNAs can be important regulators of LPS-induced innate immune response in BMDMs.

\section{Competing interests}

The authors declare that they have no competing interests.
Authors' contributions

WSW conceived the research topic and provided essential guidance. CCC performed the data collection and data processing and wrote the manuscript. All authors have read and approved the final manuscript.

\section{Acknowledgements}

This study was supported by National Cheng Kung University and Ministry of Science and Technology of Taiwan MOST-103-2221-E-006-174-MY2.

\section{Declaractions}

Publication charges of this articles was funded by National Cheng Kung University and Ministry of Science and Technology of Taiwan MOST-1032221-E-006-174-MY2.

This article has been published as part of BMC Genomics Volume 16 Supplement 12, 2015: Joint 26th Genome Informatics Workshop and 14th International Conference on Bioinformatics: Genomics. The full contents of the supplement are available online at http://www.biomedcentral.com/ bmcgenomics/supplements/16/S12.

Published: 9 December 2015

\section{References}

1. He X, Jia H, Jing Z, Liu D: Recognition of pathogen-associated nucleic acids by endosomal nucleic acid-sensing toll-like receptors. Acta Biochim Biophys Sin (Shanghai) 2013, 45(4):241-258. 
2. O'Neill LAJ: When signaling pathways collide: positive and negative regulation of toll-like receptor signal transduction. Immunity 2008, 29(1):12-20.

3. Kondo T, Kawai T, Akira S: Dissecting negative regulation of toll-like receptor signaling. Trends Immunol 2012, 33(9):449-458.

4. O'Neill LA, Sheedy FJ, McCoy CE: Micrornas: the fine-tuners of toll-like receptor signalling. Nat Rev Immunol 2011, 11(3):163-175.

5. Alam MM, O'Neill LA: Micrornas and the resolution phase of inflammation in macrophages. Eur J Immunol 2011, 41(9):2482-2485.

6. Contreras J, Rao DS: Micrornas in inflammation and immune responses. Leukemia 2012, 26(3):404-413.

7. Dai R, Ahmed SA: Microrna, a new paradigm for understanding immunoregulation, inflammation, and autoimmune diseases. Trans/ Res 2011, 157(4):163-179.

8. Xiao C, Rajewsky K: Microrna control in the immune system: basic principles. Cell 2009, 136(1):26-36.

9. Liu G, Abraham E: Micrornas in immune response and macrophage polarization. Arterioscler Thromb Vasc Biol 2013, 33(2):170-177, doi:10.1161/ ATVBAHA.112.300068.

10. Barrett T, Wilhite SE, Ledoux P, Evangelista C, Kim IF, Tomashevsky M, Marshall KA, Phillippy KH, Sherman PM, Holko M, Yefanov A, Lee H, Zhang N, Robertson CL, Serova N, Davis S, Soboleva A: Ncbi geo: archive for functional genomics data sets-update. Nucleic Acids Res 2013, 41(Database issue):991-995.

11. Barish GD, Yu RT, Karunasiri M, Ocampo CB, Dixon J, Benner C, Dent AL, Tangirala RK, Evans RM: Bcl-6 and nf-kappab cistromes mediate opposing regulation of the innate immune response. Genes Dev 2010, 24(24):2760-2765.

12. El Chartouni C, Rehli M: Comprehensive analysis of tlr4-induced transcriptional responses in interleukin 4-primed mouse macrophages. Immunobiology 2010, 215(9-10):780-787.

13. Ghigo E, Barry AO, Pretat L, Al Moussawi K, Desnues B, Capo C, Kornfeld H, Mege J-L: Il-16 promotes t. whipplei replication by inhibiting phagosome conversion and modulating macrophage activation. PLOS One 2010, 5(10):13561..

14. Mages J, Dietrich $H$, Lang R: A genome-wide analysis of Ips tolerance in macrophages. Immunobiology 2007, 212(9-10):723-737.

15. Millien VO, Lu W, Shaw J, Yuan X, Mak G, Roberts L, Song L-Z, Knight JM, Creighton CJ, Luong A, Kheradmand F, Corry DB: Cleavage of fibrinogen by proteinases elicits allergic responses through toll-like receptor 4 . Science 2013, 341(6147):792-796.

16. Eppig JT, Blake JA, Bult CJ, Kadin JA, Richardson JE, MGDG: The mouse genome database (mgd): facilitating mouse as a model for human biology and disease. Nucleic Acids Res 2015, 43(Database issue):726-736.

17. Hsu S-D, Tseng Y-T, Shrestha S, Lin Y-L, Khaleel A, Chou C-H, Chu C-F, Huang $\mathrm{H}-\mathrm{Y}$, Lin C-M, Ho S-Y, Jian T-Y, Lin F-M, Chang T-H, Weng S-L, Liao K-W, Liu C-C, Huang H-D: mirtarbase update 2014: an information resource for experimentally validated mirna-target interactions. Nucleic Acids Res 2014, 42(Database issue):78-85.

18. Maragkakis M, Reczko M, Simossis VA, Alexiou P, Papadopoulos GL, Dalamagas T, Giannopoulos G, Goumas G, Koukis E, Kourtis K, Vergoulis T, Koziris N, Sellis T, Tsanakas P, Hatzigeorgiou AG: Diana-microt web server: elucidating microrna functions through target prediction. Nucleic Acids Res 2009, 37(Web Server issue):273-276.

19. Wong $N$, Wang $X$ : mirdb: an online resource for microrna target prediction and functional annotations. Nucleic Acids Res 2015, 43(Database issue):146-152.

20. Krek A, Grün D, Poy MN, Wolf R, Rosenberg L, Epstein EJ, MacMenamin P, da Piedade I, Gunsalus KC, Stoffel M, Rajewsky N: Combinatorial microrna target predictions. Nat Genet 2005, 37(5):495-500.

21. Bolstad B, Collin F, Brettschneider J, Simpson K, Cope L, Irizarry R, Speed T: Quality assessment of affymetrix genechip data. In Bioinformatics and Computational Biology Solutions Using $R$ and Bioconductor. Volume 3. Springer, New York;Gentleman R, Carey V, Huber W, Irizarry R, Dudoit S 2005:33-47.

22. Smyth G: Bioinformatics and computational biology solutions using $r$ and bioconductor. In Bioinformatics and Computational Biology Solutions Using $R$ and Bioconductor. Volume 23. Springer, New York;Gentleman R, Carey V, Huber W, Irizarry R, Dudoit S 2005:397-420.

23. Bindea G, Galon J, Mlecnik B: Cluepedia cytoscape plugin: pathway insights using integrated experimental and in silico data. Bioinformatics 2013, 29(5):661-663.
24. Na Y-J, Kim JH: Understanding cooperativity of micrornas via microrna association networks. BMC Genomics 2013, 14(Suppl 5):17.

25. Bader GD, Hogue CW: An automated method for finding molecular complexes in large protein interaction networks. BMC Bioinformatics 2003, $4: 2$.

doi:10.1186/1471-2164-16-S12-S13

Cite this article as: Chiu and Wu: Investigation of microRNAs in mouse macrophage responses to lipopolysaccharide-stimulation by combining gene expression with microRNA-target information. BMC Genomics 2015 16(Suppl 12):S13.

\section{Submit your next manuscript to BioMed Central and take full advantage of:}

- Convenient online submission

- Thorough peer review

- No space constraints or color figure charges

- Immediate publication on acceptance

- Inclusion in PubMed, CAS, Scopus and Google Scholar

- Research which is freely available for redistribution

Submit your manuscript at www.biomedcentral.com/submit
Ciomed Central 